\title{
Novel mutant alleles of the starch synthesis gene TaSSIVb-D result in the reduction of starch granule number per chloroplast in wheat
}

Huijun Guo ${ }^{\dagger}$, Yunchuan Liư ${ }^{\dagger}$ Xiao Li, Zhihui Yan, Yongdun Xie, Hongchun Xiong, Linshu Zhao, Jiayu Gu, Shirong Zhao and Luxiang Liu*

\begin{abstract}
Background: Transient starch provides carbon and energy for plant growth, and its synthesis is regulated by the joint action of a series of enzymes. Starch synthesis IV (SSIV) is one of the important starch synthase isoforms, but its impact on wheat starch synthesis has not yet been reported due to the lack of mutant lines.

Results: Using the TILLING approach, we identified 54 mutations in the wheat gene TaSSIVb-D, with a mutation density of 1/165 Kb. Among these, three missense mutations and one nonsense mutation were predicted to have severe impacts on protein function. In the mutants, TaSSIVb-D was significantly down-regulated without compensatory increases in the homoeologous genes TaSSIVb-A and TaSSIVb-B. Altered expression of TaSSIVb-D affected granule number per chloroplast; compared with wild type, the number of chloroplasts containing 0-2 granules was significantly increased, while the number containing 3-4 granules was decreased. Photosynthesis was affected accordingly; the maximum quantum yield and yield of PSII were significantly reduced in the nonsense mutant at the heading stage.

Conclusions: These results indicate that TaSSIVb-D plays an important role in the formation of transient starch granules in wheat, which in turn impact the efficiency of photosynthesis. The mutagenized population created in this study allows the efficient identification of novel alleles of target genes and could be used as a resource for wheat functional genomics.
\end{abstract}

Keywords: Wheat, TILLING, Mutant, TaSSIVb-D, Gene expression, Starch granule

\section{Background}

Starch granules in plants are classified into two types according to their physiological role. Some starch granules are stored for long periods in reserve tissues as storage starch. This type of starch is an important source of carbohydrates for human beings. Other types of starch are stored temporarily as transient starch to provide carbon and energy for plant growth. Transient starch is synthesized in chloroplasts during the day and consumed

\footnotetext{
*Correspondence: liuluxiang@caas.cn

'Equal contributors

Institute of Crop Sciences, Chinese Academy of Agricultural Sciences/ National Key Facility for Crop Gene Resources and Genetic Improvement/ National Center of Space Mutagenesis for Crop Improvement, Beijing 100081, China
}

(c) The Author(s). 2017 Open Access This article is distributed under the terms of the Creative Commons Attribution 4.0 International License (http://creativecommons.org/licenses/by/4.0/), which permits unrestricted use, distribution, and reproduction in any medium, provided you give appropriate credit to the original author(s) and the source, provide a link to the Creative Commons license, and indicate if changes were made. The Creative Commons Public Domain Dedication waiver (http://creativecommons.org/publicdomain/zero/1.0/) applies to the data made available in this article, unless otherwise stated. in the plastid stroma by joint action of ADP-glucose pyrophosphorylases (AGPase), starch synthases (SS), starch branching enzymes (SBE), starch debranching enzymes (DBE) and their isoforms; however, the synthesis and regulation of transient granules hold a specific feature [1]. Some synthase isoforms that synthesize storage starch are different from those that synthesize transient starch.

AGPase is the first and rate-limiting enzyme in the biosynthesis of starch, it catalyzes the synthesis of ADPglucose, which is the substrate of amylose starch, and over expression of the AGPase gene enhances the rate of starch biosynthesis [2-4]. Amylose, one of the main 
forms of starch, is synthesized from the substrate, ADPglucose, by granule-bound starch synthase (GBSS). The isoform GBSS2 is responsible for amylose synthesis in leaves and other non-storage tissues [5-8], whereas amylopectin, the other major form of starch, is synthesized by the coordinated actions of AGPase, SS, and SBE. Five soluble SS isozymes, SSI, SSII, SSIII, SSIV, and SSV are found in plant genomes [9, 10]; and among them, SSI, SSII, and SSIII are involved in amylopectin elongation [11]. Isoform SSI is highly expressed in plant leaves [12, 13]. Two or three isoforms of SSII and SSIII are found in the genomes of different species, and at least one isoform is expressed in leaves and regulates starch granule biosynthesis [14-17]. Only one SSIV isoform is found in the Arabidopsis genome; AtSSIV is involved in the initiation of starch granule formation in leaves [18, 19]. Overexpression of AtSSIV increases the levels of starch accumulation by $30-40 \%$ and results in a higher rate of growth [20]; SSIV mutants have remarkably decreased numbers of granules and abnormal granules $[18,19,21]$.

The functions of SSIV isozymes in the leaves of cereal crops are not as clear as in Arabidopsis. Two SSIV isoforms, SSIVa and SSIVb, are found in rice. SSIVa is mainly expressed in the endosperm and is responsible for starch accumulation in seeds, whereas SSIVb is mainly expressed in leaves at early development stages and is responsible for leaf granule biosynthesis [12, 14, 22]. It also has overlapping and crucial roles with SSIIIa in rice seeds in determining granule morphology and in maintaining the amyloplast envelope structure [23]. $Z m S S I V$ is highly expressed in the embryo, endosperm and pericarp in maize. In wheat, only one isoform of SSIV has been identified until now. This gene is located on the long chromosome arm of homologous group I and shows high similarity with OsSSIVb, so this isoform is named TaSSIVb [12, 24]. TaSSIVb is preferentially expressed in leaves and is not regulated by the circadian clock [24]. TaSSIVb is also highly expressed at the middle stages of seed development $[25,26]$; however, the impact of TaSSIVb on wheat leaf and seed granule characteristics at different growth stages have not yet been reported due to the lack of mutant lines.

TILLING (Targeting-Induced Local Lesions IN Genomes) is a powerful approach for novel allele identification and has been used to screen mutagenized plant populations for novel alleles of target genes [27-34]. Hundreds of missense and nonsense mutant alleles of key starch synthesis-related wheat genes, such as TaSSII, TaSBEIIa, TaSBEIIb, have been identified using this platform $[35,36]$. A hexaploid wheat line containing two waxy homozygous mutations created through TILLING and a pre-existing deletion of a third waxy homoeolog displays a near-null phenotype [37]. Novel alleles of
TaSBEII have been identified in both durum wheat and bread wheat. Double mutant lines combining a TaSBEIIa-A mutation with a TaSBEIIa-B mutation in durum wheat and triple mutant lines combining mutations in TaSBEIIa-A, TaSBEIIa-B with TaSBEIIa-D have a significant increase in both amylose and resistant starch content [38-42]. Using this approach to identify novel alleles of TaSSIVb would be helpful in characterizing its role in transient starch granule synthesis in wheat leaves.

In this paper, we used the TILLING approach to identify novel alleles of TaSSIVb-D with mutations in functional regions in an Ethyl methanesulphonate (EMS) mutagenized population derived from an elite hexaploid wheat cultivar. Homozygous mutants were used to investigate the specific role of TaSSIVb-D in starch granule synthesis in leaves at different stages of growth. We found that TaSSIVb-D mutations decrease gene expression and the number of starch granules in leaves.

\section{Results}

Novel alleles of TaSSIVb-D in a mutagenized population

Three primer sets corresponding to TaSSIVb-D were designed for allele mining (Table 1), and their specificities were validated using nullisomic-tetrasomic lines (Fig. 1a). The location of these primers on the TaSSIVb-D sequence is shown in Fig. 1b.

In the mutagenised population, 54 mutations were identified with a mutation density of $1 / 165 \mathrm{~Kb}$. Among these mutations, 52 are $\mathrm{G}$ to $\mathrm{A}(61.12 \%)$ or $\mathrm{C}$ to $\mathrm{T}$ (35.19\%) transition point mutations, and the other 2 are $\mathrm{T}$ insertions. Of these mutations, 26 are in the coding region (Table 2), including 1 nonsense mutation (E05413), 15 missense mutations and 10 silent mutations. The remaining 26 are located in introns and include 2 splice junction mutations.

Using SIFT program, five missense mutations (E2-2226 and E2-2-437, E2-2-193, E570, E972, E1137) are predicted to have a severe impact on protein function. Based on the PSSM program, three missense mutations (E2-2-226 and E2-2-437, E972, E1137) are predicted to have a severe impact. As the nonsense mutant E054-13 results in the loss of both the starch catalytic domain and glycosyltranferase domain, it might have a severe impact on protein function. Because the E1137 mutation is located in the glycosyltranferase domain which has less similarity with other SS isoforms [24], and might have distinct functions from other starch synthases,

Table 1 Primer sequences used for allele mining

\begin{tabular}{lll}
\hline Name & Forward $\left(5^{\prime}-3^{\prime}\right)$ & Reverse $\left(5^{\prime}-3^{\prime}\right)$ \\
\hline s4b-D1 & ACTAAAACCCACTTGGGAC & GGTAGGAATGATACAGAACACC \\
s4b-D2 & CTGCAAAAAATTGTCTAAAAGCTAC & CATGCTTGAAATTATCTACTTTCG \\
s4b-D3 & ACCAGAAATTCAGGTGCGTT & TGAGTCGTGTTGTGCCCG \\
\hline
\end{tabular}




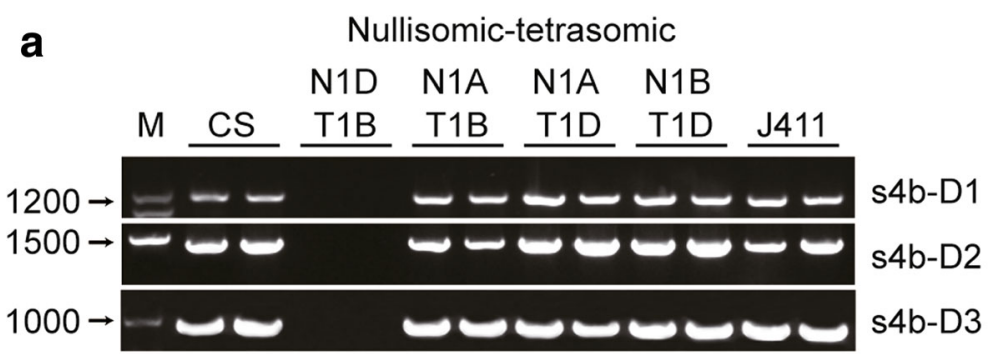

b

s4b-D1F s4b-D1R s4b-D2F s4b-D2R s4b-D3F s4b-D3R

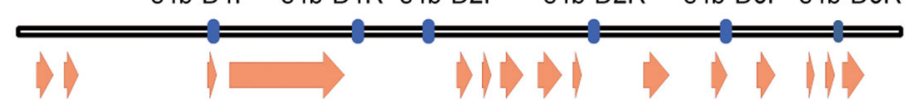

Fig. 1 Validation of subgenome-specific TaSSIVb-D primers using the Chinese Spring nullisomic-tetrasomic lines. a Products from the Chinese Spring (CS), nullisomic-tetrasomic lines (N1AT1B, N1AT1D, N1BT1D and N1DT1B) and wild type (J411) amplified using the three different sets of TaSSIVb-D-specific primer pairs shown in b. TaSSIVb-D is absent from N1DT1B. b Diagram of D subgenome primers. The black rectangle represents the gene sequence; orange arrowheads indicate exon regions; blue rectangles represent the location of each primer

Table 2 Mutations identified in the coding region of the TaSSIV b-D gene

\begin{tabular}{|c|c|c|c|c|c|}
\hline Line & Allele & Mutation Type & Effect & PSSM & SIFT \\
\hline E334 & G1783A & Silent & & & \\
\hline E1239 & G1813A & Missense & E181K & 4.4 & 0.6 \\
\hline E469 & G1822A & Missense & E183K & -6 & 0.42 \\
\hline E939 & G1848A & Silent & & & \\
\hline E1337 & G1863A & Silent & & & \\
\hline E2-2-226 & T1880C & Missense & L203S & 26.7 & 0 \\
\hline E2-2-437 & T1880C & Missense & L203S & 26.7 & 0 \\
\hline E468 & G1890A & Silent & & & \\
\hline E1245 & G1920A & Silent & & & \\
\hline E391 & G1975A & Missense & D235N & & \\
\hline E137 & G2008A & Missense & $\mathrm{D} 246 \mathrm{~N}$ & 0.4 & 0.96 \\
\hline E054-13 & C2077T & Nonsense & Q269-Stop & & \\
\hline E2-2-193 & C2143T & Missense & L291F & 0.6 & 0.02 \\
\hline E2-2-95 & C2152T & Missense & L294F & & \\
\hline E570 & A2219G & Missense & Q316R & 8.4 & 0 \\
\hline E1347 & C2353T & Silent & & & \\
\hline E783 & G2402A & Missense & S377N & & \\
\hline E236 & G2413A & Silent & & & \\
\hline E996 & C3516T & Silent & & & \\
\hline E2-2-181 & G3536A & Missense & V469| & & \\
\hline E054-9 & C3740T & Intronic & & & \\
\hline E1226 & G3932A & Missense & V550I & 0.9 & 0.09 \\
\hline E972 & С3950T & Missense & $\mathrm{H} 556 \mathrm{Y}$ & 27.5 & 0 \\
\hline E930 & G5872A & Silent & & & \\
\hline E1373 & C5878T & Silent & & & \\
\hline E1137 & G6327A & Missense & S833N & 12 & 0 \\
\hline
\end{tabular}

Nucleotide changes are numbered relative to the start codon ATG mutants E1137 and E054-13 were selected for analysis of gene expression and starch granule number.

\section{The expression of TaSSIVb in mutant leaves at different growth stages}

Specific primer sets for each sub-genome (Additional file 1: Table S1, Additional file 2: Figure S1) were used to analyze the pattern of TaSSIVb gene expression. In wild type, gene expression profiling revealed non-significant differences in expression between different growth stages (Fig. 2). In the mutants, TaSSIVb-D was down-regulated, and TaSSIVb-A and TaSSIVb-B did not show significant compensatory responses. The expression of TaSSIVb-D in the nonsense mutant E054-13 was reduced by $\sim 8$-fold compared with the wild type at all three growth stages, whereas in E1137, its expression level gradually increased with plant growth and only showed a significant reduction (36.12\%) at the seedling stage (Fig. 2a). In contrast, no significant differences in the expression of TaSSIVb-A (Fig. 2b) and TaS$S I V b-B$ (Fig. 2c), were detected in the two TaSSIVb-D mutants at all three stages, except for TaSSIVb-A expression in the E054-13 mutant at the seedling stage (9.55\% reduction).

\section{Variation in the number of starch granules in the chloroplast}

We investigated starch granule characteristics in the chloroplasts of wild type and the two mutants at three growth stages (Fig. 3). Granule number varied plast by plast in each genotype, with numbers ranging from 0 to 8 and more than $90 \%$ of chloroplasts contained no more than 4 granules (Additional file 1: Table S2). The difference between the wild type and mutants was mainly in the number of chloroplasts containing no more than 4 granules. 

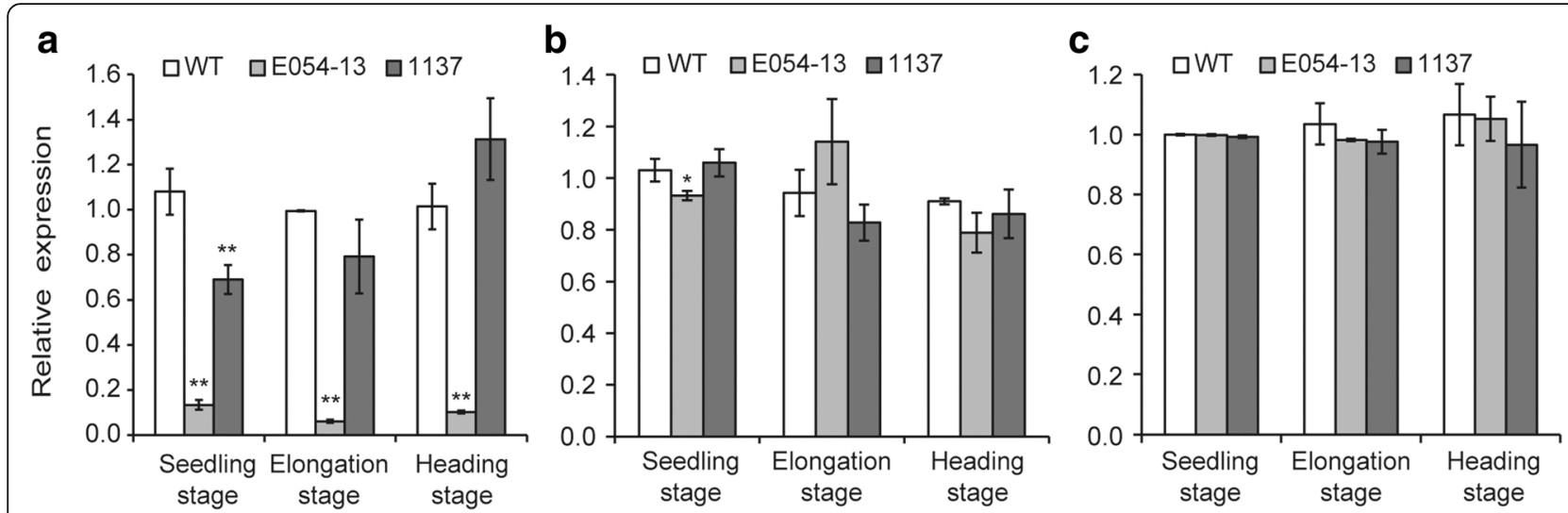

Fig. 2 Expression patterns of the three homeologous TaSSIVb genes in leaves at different stages. a Relative expression level of TaSSIVb-D;

b Relative expression level of TaSSIVb-A; c Relative expression level of TaSSIVb-B. Error bars represent standard deviation; WT: wild type; * means significantly different from WT at the $P=0.05$ level and ${ }^{* *}$ means significantly different from WT at the $P=0.01$ level based on Student's $t$ test

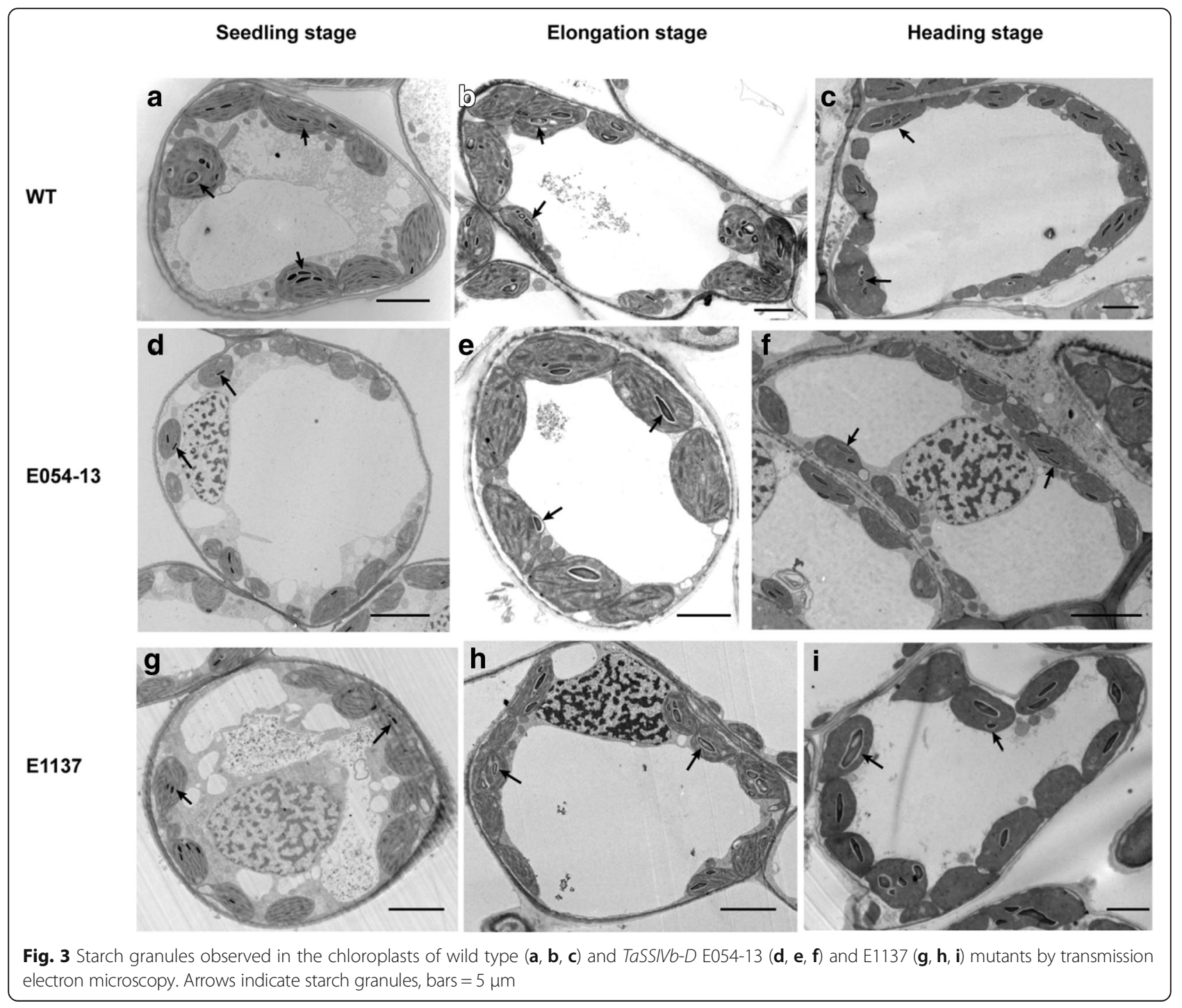


The biggest difference in granule number per chloroplast was observed at the seedling stage, and mutation of $T a S S I V b-D$ led to a reduction in granule number. The percentage of chloroplasts with $0-2$ granules in both mutants, E054-13 (96.70\%) and E1137 (84.70\%), were significantly higher compared with wild type (70.30\%) (Fig. 4), whereas at the elongation and heading stages, the difference between E1137 and wild type became smaller; only the E054-13 mutant had a significantly higher percentage of chloroplasts with $0-2$ and 3-4 granules $(17.74 \%$ and $18.72 \%$ higher than wild type, respectively). In contrast, the percentage of chloroplasts containing 3-4 granules was lower in the mutants than in wild type. At the seedling stage, the percentage of chlorplasts in E054-13 and E1137 with 3-4 granules was $22.00 \%$ and $10.3 \%$ lower than wild type, respectively. At the elongation and heading stages, only the E054-13 mutant was significantly different from wild type; the percentage of chlorplasts with 3-4 granules was $14.26 \%$ and $15.05 \%$ lower than wild type, respectively. The observation of the chloroplast granule number phenotype at all stages for the E054-13 mutant and only at the seedling stage for the E1137 mutant is consistent with the lower levels of TaSSIVb-D gene expression in the mutants at these stages (Fig. 2).

\section{Photosynthesis parameters and the efficiency of PSII}

As transient starch in chloroplasts provides carbon and energy for plant growth, we deduced that photosynthesis might be affected by reduced granule numbers. Granule numbers in the mutant E054-13 were more severely reduced than those in E1137, and the impact on photosynthesis parameters are expected to be more severe. Therefore, photosynthesis parameters in the mutant E054-13 and the wild type at the heading stage were compared to determine the impact of reduced granule number. We found that the maximum quantum yield $\left(F_{v} / F_{m}\right)$ and yield of PSII (Y(II)) of E054-13 were significantly lower than in wild type (Table 3), and differences between the mutant and wild type in both Y(II) and electron transport rate (ETR) increased with increasing photosynthetically active radiation (PAR) (Fig. 5). As Y(II) provides the effective quantum yield and could be used to estimate the effective portion of absorbed quanta; while ETR provides information for plant stress reaction, so the results indicates that mutation of TaS$S I V b-D$ leads to a decrease in relative quantum yield and has a negative effect on PSII efficiency.

\section{Discussion}

\section{Conserved and functional regions of TaSSIVb}

As one of the isoforms of the soluble starch synthesis family, wheat SSIV contains the starch catalytic domain (GT-5) and glycosyltranferase domain (GT-1). GT-5 (amino acids 422-661) is more conserved than GT-1 (amino acids 706-886) between SS isoforms [24]. Two mutations that we identified in this study, E972 and E1137, are located in GT-5 and GT-1, respectively, and in the wild type these amino acids are involved in the formation of $\beta$-sheets (data not shown). In contrast the $\mathrm{N}$-terminus (amino acids 1-405) is distinct from other SS isoforms, and this region contains two coiled-coil domains and a 14-3-3-protein binding site [24]. The missense mutation E2-2-226 and the truncation mutation E054-13 are located in this region. These novel alleles could be very useful in understanding the specific functions of SSIV in starch granule synthesis in wheat. The gene expression patterns and alteration of granule number in the E1137 and E054-13 mutants demonstrate the important roles of these predicted functional regions. The E054-13 truncation mutation has a more severe effect than the missense mutation;
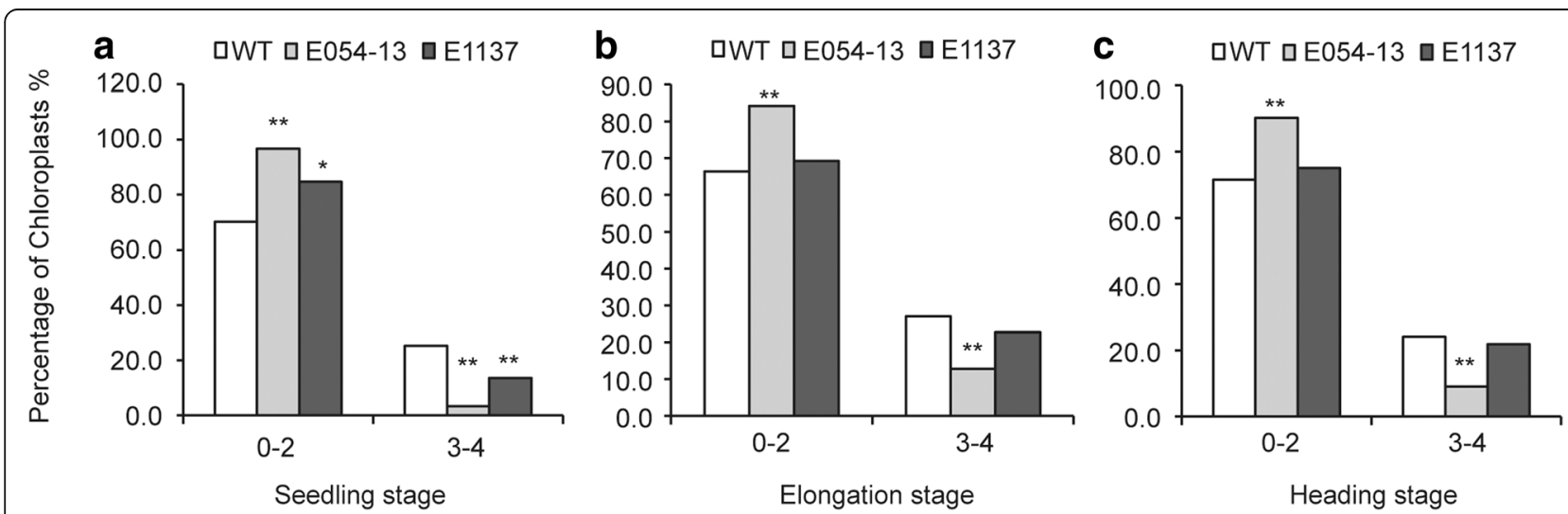

Fig. 4 Percentage of chloroplasts in wild type (WT) and TaSSIVb-D mutants (E054-13 and E1137) containing 0-2 and 3-4 granules at three different growth stages. a Seedling stage; $\mathbf{b}$ Elongation stage; $\mathbf{c}$ Heading stage. The percentage of chloroplasts equals the number chloroplasts containing $0-2$ and 3-4 starch granules divided by the total number of chloroplasts observed. ${ }^{*}$ means significantly different from WT at the $P=$ 0.05 level and ** means significantly different from WT at the $P=0.01$ level 
Table 3 Changes in chlorophyll fluorescence parameters in the TaSSIVb-D E054-13 nonsense mutant at the heading stage

\begin{tabular}{lll}
\hline Genotype & $Y(I I)$ & $F_{v} / F_{m}$ \\
\hline Wild type & $0.641 \pm 0.0019$ & $0.818 \pm 0.0175$ \\
E054-13 & $0.617 \pm 0.0020 * *$ & $0.622 \pm 0.0274 * *$ \\
\hline
\end{tabular}

$\mathrm{Y}(\mathrm{II})$ and $F_{\mathrm{v}} / F_{m}$ were measured in dark-adapted plants, and Photosynthetically Active Radiation (PAR) equals 281 . Values are means \pm standard deviation ** significantly different from the wild type at the $P<0.01$ level based on Student's $t$ test

TaSSIVb gene expression is reduced by $5 \sim 13$-fold compared with E1137 (Fig. 2), and granule number is also significantly decreased compared with E1137 (Additional file 1: Table S2).

\section{Expression of TaSSIVb homoeologs}

Wheat has three sub-genomes, and the literature shows that the contribution of each homeologous gene can be different. For example, of the three SSIIa genes, SSIIa on the $\mathrm{B}$ genome has the largest contribution to amylopectin structure than those on the other two genomes [43]. Each waxy gene encoding GBSSI has different effects on amylopecitin characteristics [37, 44, 45]. SS isoform SSIV is encoded by three homeologous genes located on group I chromosomes [24] and the effect of each gene and their interactions is not clear. Here we designed homeologous-specific primers to determine the gene expression patterns of the three SSIV genes. In TaSSIVb-D mutants, decreased expression of TaSSIVb-D did not result in significant changes in the expression of TaSSIVb$A$ or TaSSIVb-B. This is similar to what was observed in the single null mutant of SBEIIa; a mutation in one subgenome does not result in appreciably different expression in the other two sub-genomes [46]. However, the identification of mutations in TaSSIVb-A and TaSSIVb-B is needed to analyze the interaction between homoeologous copies or their dosage effects.

\section{Expression of TaSSIV-D in wheat leaves}

The function of AtSSIV, which it is highly expressed in leaves in Arabidopsis is very well understood. In wheat, the expression of TaSSIVb is tissue-dependent, and is much more highly expressed in leaves than in the endosperm [24]. Our results are consistent with these reports; all three homeologous genes are highly expressed in leaves during the entire growth period. There is also evidence that TaSSIVb is expressed during seed development [24-26]. In ongoing research we are investigating the accumulation of storage starch in both the E054-13 and E1137 mutants to elucidate the role of TaSSIVb in storage starch synthesis.

\section{Starch granule synthesis}

Starch granules are initiated early in the development of young leaves in Arabidopsis. Because more starch granules are observed in immature leaves than in mature leaves [47], it is believed that SSIV functions in granule initiation at earlier growth periods [21]. The $s s I V$ mutant in Arabidopsis has reduced granules in most chloroplasts $[18,21]$. The results of this study demonstrate that in young wheat leaves TaSSIV-D has a function similar to AtSSIV, and it is involved in initiation of transitory starch synthesis. At the seedling stage, the expression patterns of both the E054-13 and E1137 TaSSIV-D mutants are down regulated. In the E1137 mutant, the significant reduction of granule number is only observed when the expression of TaSSIV-D is reduced; while in the nonsense mutant, starch granules are reduced at all the three stages. This indicates that reduction of starch granule number results from the mutation of TaSSIV-D and not other genes.

\section{Effect of homeologous genes}

In many cases, homeologous genes from different subgenomes of wheat have dosage effects on phenotypes. Only double or triple mutants of SSIIa have severe
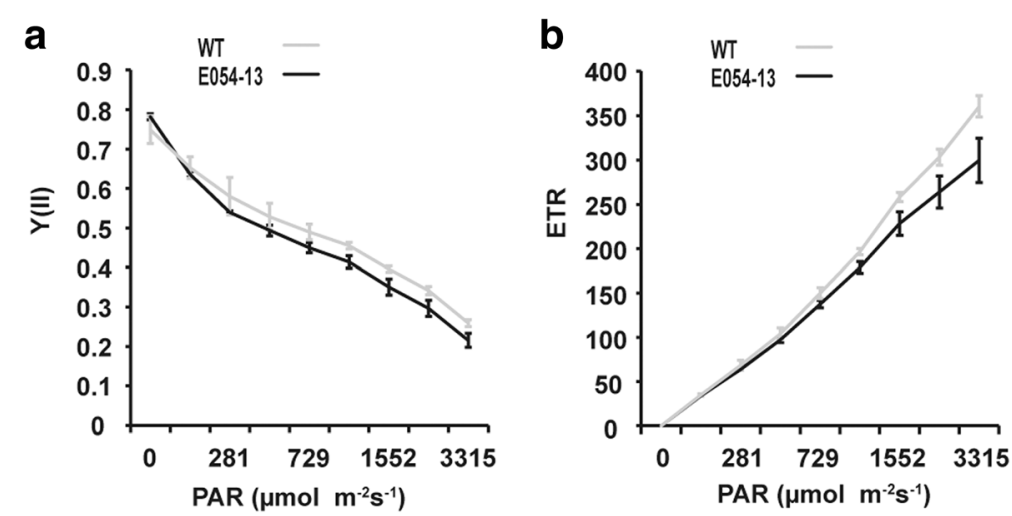

Fig. 5 Y (II) (a) and ETR (b) fast light curves for wild type and the TaSSIVb-D E054-13 nonsense mutant at the heading stage. Error bars represent standard deviation 
effects on starch properties $[48,49]$, and single-gene mutants do not show significant phenotypes [38]. However in single TaSSIV-D gene mutants, gene expression level, granule number, relative quantum yield and PSII efficiency are significantly reduced with respect to the wild type. It has been reported that single mutants of the genes SBEIIa and gpc (grain protein content) also show significant phenotypic differences compared with the wild type $[46,50]$, so not only double and triple mutants, but also single mutants can lead to phenotypic changes in hexaploid wheat.

\section{Relationship between transient starch content and photosynthesis}

It has been reported that the capacity for starch synthesis and the rate of photosynthesis are positively correlated [51]. A starchless tobacco mutant shows about $50 \%$ inhibition of maximum photosynthetic capacity [52]. Mutation of SSIV in Arabidopsis also has negative effects on photosynthesis efficiency [53]. Our SSIV mutant in wheat shows the same effects; photosynthesis efficiency is significantly reduced. This is likely because SSIV mutants accumulate high concentrations of the substrate ADPglucose [21], which leads to photooxidative stress and decreased photosynthesis efficiency [53].

\section{Conclusions}

We identified novel alleles, including missense and nonsense mutations, of the starch synthesis gene, TaSSIVb$D$. Gene expression, granule number and photosynthetic parameters are down-regulated or reduced in the missense mutant E1137 and the nonsense mutant E054-13, and the impact of E054-13 is much more severe than that of E1137. This demonstrates TaSSIVb-D plays an important role in the formation of transient starch granules in wheat, and mutation of TaSSIVb-D results in remarkable reduction in transitory starch granules in leavies. The mutagenized population generated in this study could be used as a resource for gene functional research.

\section{Methods}

\section{Materials}

Wheat (Triticum aestivum L.) cultivar Jing411 was used as the wild type (WT) to create the mutagenized population. Homozygous mutant lines grown in the field with normal management were used for RT-qPCR and phenotyping.

\section{Development of the wheat TILLING population}

Seeds from the wild type Jing411 were soaked in tap water for $10 \mathrm{~h}$ at $20^{\circ} \mathrm{C}$, then incubated in $1.5 \%$ EMS in $0.1 \mathrm{M}$ sodium phosphate buffer ( $\mathrm{pH} 7.0)$ for $4 \mathrm{~h}$ or $6 \mathrm{~h}$ at $20^{\circ} \mathrm{C}$. This was followed by washing with running tap water for $4 \mathrm{~h}$ at room temperature. The mutagenized seeds $\left(M_{1}\right.$ generation) were planted directly in the field and self-fertilized. The $\mathrm{M}_{2}$ was developed using the single-seed descent (SSD) method.

The mutagenized population consists of $3058 \mathrm{M}_{2}$ plants, including 1441 individuals from the $4 \mathrm{~h} 1.5 \%$ EMS treatment and 1617 from the $6 \mathrm{~h}$ treatment. A young leaf from each individual was sampled at the seedling stage for genomic DNA extraction using the DNA-quick Plant System kit (Tiangen Biotech, Beijing, China). DNA concentration was measured with the NanoDrop2000 spectrophotometer (Thermo Fisher Scientific, USA) according to the manufacturer's instructions, and the final concentration was adjusted to $50 \mathrm{ng} /$ $\mu \mathrm{l}$. Individual DNA samples were pooled 2-fold into 96well plates for TILLING screening, which was done according to Till et al. [54].

\section{Primer design}

The sequence of TaSSIVb (DQ400416) was downloaded fromhttps://www.ncbi.nlm.nih.gov/nuccore/DQ400416, and the sequences of TaSSIVb-A, TaSSIVb-B and TaSSIVb-D were obtained through alignment with long arm of chromosome 1A, 1B and 1D (https://wheat-urgi.versailles.inra.fr/ Seq-Repository). TaSSIVb-D was used as the target gene for mining and phenotyping novel alleles. Primer Premier 5.0 (Premier Biosoft International, Palo Alto, CA) was used to design homoeolog-specific TILLING and qPCR primers (Table 1 and Additional file 1: Table S1), and their specificities were validated using Chinese Spring nullisomictetrasomic lines (N1AT1B, N1AT1D, N1BT1D and N1DT1B; Fig. 1a and Additional file 2: Figure S1).

\section{Mutation screening}

Each primer used for TILLING detection was synthesized with and without fluorescent dye label. Unlabeled primers (Sangon Biotech, China), forward primers labeled with the fluorescent dye IRD700 and reverse primers labeled with the fluorescent dye IRD800 (Bioneer, China) were mixed with the ratio of unlabeled forward: labeled forward: unlabeled reverse: labeled reverse $=4: 1: 1: 4$. PCR amplification and polyacrylamide gel electrophoresis were carried out as previously described [54]. Identified mutations were sequenced to verify nucleotide variation. Mutation density was calculated by dividing the total number of mutations by the total length of the sampled DNA sequence (length of the amplified fragment $\times$ number of individuals sampled). Totally 3058 individual plants were screened for each primer set, and the amplicon size of primer set s4bD1, s4b-D1 and s4b-D1 was 1191, 1361 and 960 bp respectively. As DNA products at the top and bottom of the gel were difficult to detect, we subtracted $100 \mathrm{bp}$ from the 5 terminus and $100 \mathrm{bp}$ from the 3 ' terminus of each fragment. 
The PARSESNP (Project Aligned Related Sequences and Evaluate SNPs; http://blocks.fhcrc.org/proweb/parsesnp/) and SIFT (Sorting Intolerant from Tolerant; http://sift. bii.a-star.edu.sg/) programs were used to predict the severity of each mutation. Mutations with PSSM $>10$ or SIFT $<0.05$ are predicted to have a severe effect on protein function $[55,56]$.

\section{RT-qPCR}

The effects of mutations on the expression of TaSSIVb gene homoeologs was evaluated for leaves at the seedling, elongation and heading stages. Total RNA was extracted from three individual plant leaves, and cDNA was synthesized using the FastQuant RT kit (With gDNase, Tiangen Biotech) according to the manufacturer's instructions. RNA extraction and cDNA synthesis was done for three biological replicates per genotype with three technical replicates for each biological replicates, and their concentrations measured with the NanoDrop2000 spectrophotometer, the same volume and concentration of normalized template was used for the next step experiment. RT-qPCR was performed on a CFX96 system using the SsoFast EvaGreen Supermix kit (Bio-Rad), PCR was performed at $94^{\circ} \mathrm{C}$ for $3 \mathrm{~min}$, followed by 40 cycles at $94^{\circ} \mathrm{C}$ for $30 \mathrm{~s}$, annealing for 30 $\mathrm{s}$, and $72^{\circ} \mathrm{C}$ for $10 \mathrm{~s}$, and then a melt curve stage. Concentration of each primer was $0.3 \mu \mathrm{mol}$, amplification efficiency of the three genes ranged from $97 \%$ to $100 \%$. The actin gene used as a control was the same as described by $\mathrm{Gu}$ et al. [57]. The relative expression level was calculated with $\Delta \Delta C \mathrm{t}$ method according to the CFX96 manual, data were analyzed by one-way ANOVA using Microsoft Excel software.

\section{Electron microscopy}

At the seedling, elongation, and heading stages, leaves of mutants and WT at the same developmental stage were sampled with 3 replicates for transmission electron microscope analysis. Each sample was processed as described in Guo et al. [58] and photographed with a transmission electron microscope (HT-7700, Hitachi, Japan). Starch granule numbers per chloroplast were calculated, and data were analyzed using the SPSS software [59].

\section{Chlorophyll fluorescence parameters}

A pulse amplitude modulation fluorometer (MINI-PAM, Heinz Walz, Effeltrich, Germany) was used to measure chlorophyll fluorescence parameters. At the seedling, elongation, and heading stages, individual plants at the same developmental stage were selected for measurements with three biological and three technical replicates. From 10:00-11:00 am on the same day, after dark adaption by Dark Leaf Clip for $30 \mathrm{~min}$, the value of $F_{v} /$
$F_{m}$ and Y(II) were measured with a photosynthetically active radiation (PAR) of $281 \mu \mathrm{mol} \mathrm{m} \mathrm{s}^{-2} \mathrm{~s}^{-1}$ according to the manufacturer's instructions. The response of photosynthetic fluorescence parameters to light intensity changes was determined using rapid light curves. Imaging Win software (Heinz Walz, Effeltrich, Germany) was used to collect and analyze fluorescence parameter data. Data were analyzed by one-way ANOVA with Microsoft Excel software.

\section{Additional files}

Additional file 1: Table S1. Primers used for RT-qPCR. Table S2. Percentage of chloroplasts containing different numbers of starch granules. (DOCX 19 kb)

Additional file 2: Figure S1. Validation of sub-genome-specific RT-qPCR primers using Chinese Spring nullisomic-tetrasomic lines. CS: Chinese Spring; N1DT1B, N1AT1B, N1AT1D, and N1BT1D: Chinese Spring nullisomictetrasomic lines; J411: wild type; s4b-qD: TaSSIVb-D-specific primers; s4b-qA: TaSSIVb-A-specific primers; s4b-qB: TaSSIVb-B-specific primers. (DOCX 1759 kb)

\section{Abbreviations \\ AGPase: ADP-glucose pyrophosphorylases; DBE: Starch debranching enzymes; EMS: Ethyl methanesulphonate; ETR: Electron transport rate; GBSS: Granule-bound starch synthase; gpc: Grain protein content; GT- 1: Glycosyltranferase domain; GT-5: Starch catalytic domain; \\ PAR: Photosynthetically active radiation; PARSESNP: Project aligned related sequences and evaluate SNPs; RT-qPCR: Reverse transcription quantitative PCR; SBE: Starch branching enzymes; SIFT: Sorting intolerant from tolerant; SPSS: Statistical package for the social sciences; SS: Starch synthases; SSD: Single-seed descent; SSIV: Starch synthesis IV; TILLING: Targeting induced local lesions in genomes; WT: Wild type; Y(II): Yield of PSII}

\section{Acknowledgements}

We thank Dr. Xianchun Xia, Institute of Crop Science, Chinese Academy of Agricultural Sciences, Beijing, China, for kindly providing us with the nullisomic-tetrasomic lines.

\section{Funding}

This work was supported by the National Key Research and Development Program (2016YFD0102101 and 2016YFD0102106) and NSFC project (31100610) of P. R. China. The funding bodies had no role in the design of the study and collection, analysis, and interpretation of data.

Availability of data and materials

All data generated or analysed during this study are included in this publishied article and its supplementary information files. The raw data are available from the cooresponding author on resonable request.

\section{Authors' contributions}

HG and ZY developed the TILLING population and wrote the manuscript. XL screened the alleles. $Y L$ and $X L$ designed the specific primers. $Y L$ carried out the RT-qPCR and microscopy analysis. HG and $Y L$ measured chlorophyll fluorescence parameters and prepared the drafting manuscript. $Y X$ and $H X$ prepared figures. LZ, JG and SZ managed plant materials. LL conceived the original research, supervised data generation and analyses and wrote the manuscript. All authors read and approved the final muanuscript.

Competing interests

The authors declare that they have no competing interests.

Consent for publication

Not applicable. 


\section{Ethics approval and consent to participate}

Wheat samples have been used in the article, and seeds of the wild type were deposited in Institute of Crop Science, Chinese Academy of Agricultural Sciences. The mutants were developed by the authors. All plants were grown and conducted in accordance with the local legislation. No voucher specimen was deposited in public herbarium.

\section{Publisher's Note}

Springer Nature remains neutral with regard to jurisdictional claims in published maps and institutional affiliations.

\section{Received: 13 February 2017 Accepted: 25 April 2017} Published online: 08 May 2017

\section{References}

1. Nakamura Y. Starch. 2015. p. 211-37.

2. Kang G, Liu G, Peng X, Wei L, Wang C, Zhu Y, Ma Y, Jiang Y, Guo T. Increasing the starch content and grain weight of common wheat by overexpression of the cytosolic AGPase large subunit gene. Plant Physiol Biochem. 2013;73:93-8

3. Li N, Zhang S, Zhao Y, Li B, Zhang J. Over-expression of AGPase genes enhances seed weight and starch content in transgenic maize. Planta. 2011; 233(2):241-50

4. Smidansky ED, Clancy M, Meyer FD, Lanning SP, Blake NK, Talbert LE, Giroux MJ. Enhanced ADP-glucose pyrophosphorylase activity in wheat endosperm increases seed yield. Proc Natl Acad Sci U S A. 2002;99(3):1724-9.

5. Nagai YS, Sakulsingharoj C, Edwards GE, Satoh H, Greene TW, Blakeslee B, Okita TW. Control of starch synthesis in cereals: metabolite analysis of transgenic rice expressing an up-regulated cytoplasmic ADP-glucose pyrophosphorylase in developing seeds. Plant Cell Physiol. 2009;50(3):635-43.

6. Nakamura T, Vrinten P, Hayakawa K, Ikeda J. Characterization of a granulebound starch synthase isoform found in the pericarp of wheat. Plant Physiol. 1998;118:451-9.

7. Vrinten P, Nakamura T. Wheat granule-bound starch synthase I and II are encoded by separate genes that are expressed in different tissues. Plant Physiol. 2000;122:255-64.

8. Edwards A, Borthakur A, Bornemann S, Venail J, Denyer K, Waite D, Fulton D, Smith A, Martin C. Specificity of starch synthase isoforms from potato. Eur J Biochem. 1999;266:724-36.

9. Bahaji A, Li J, Sanchez-Lopez AM, Baroja-Fernandez E, Munoz FJ, Ovecka M, Almagro G, Montero M, Ezquer I, Etxeberria E, et al. Starch biosynthesis, its regulation and biotechnological approaches to improve crop yields. Biotechnol Adv. 2014;32(1):87-106.

10. Liu H, Yu G, Wei B, Wang Y, Zhang J, Hu Y, Liu Y, Yu G, Zhang H, Huang Y. Identification and phylogenetic analysis of a novel starch synthase in maize. Front Plant Sci. 2015;6:1013.

11. Luo J, Ahmed R, Kosar-Hashemi B, Larroque O, Butardo Jr VM, Tanner GJ, Colgrave ML, Upadhyaya NM, Tetlow IJ, Emes MJ, et al. The different effects of starch synthase lla mutations or variation on endosperm amylose content of barley, wheat and rice are determined by the distribution of starch synthase I and starch branching enzyme IIb between the starch granule and amyloplast stroma. Theor Appl Genet. 2015;128(7):1407-19.

12. Hirose T, Terao T. A comprehensive expression analysis of the starch synthase gene family in rice (Oryza sativa L.). Planta. 2004;220(1):9-16.

13. Kossmann J, Abel GJW, Springer F, Lloyd JR, Willmitzer L. Cloning and functional analysis of a CDNA encoding a starch synthase from potato (Solanum tuberosum L.) that is predominantly expressed in leaf tissue. Planta. 1999;208:503-11.

14. Dian W, Jiang H, Wu P. Evolution and expression analysis of starch synthase III and IV in rice. J Exp Bot. 2005;56(412):623-32.

15. Harn C, Knight M, Ramakrishnan A, Guan H, Keeling PL, Wasserman BP. Isolation and characterization of the zSSIla and zSSIlb starch synthase CDNA clones from maize endosperm. Plant Mol Biol. 1998;37:639-49.

16. Jiang H, Dian W, Liu F, Wu P. Molecular cloning and expression analysis of three genes encoding starch synthase II in rice. Planta. 2004;218(6):1062-70.

17. Zhang X, Myers AM, James MG. Mutations affecting starch synthase III in Arabidopsis alter leaf starch structure and increase the rate of starch synthesis. Plant Physiol. 2005;138(2):663-74.

18. Roldan I, Wattebled F, Mercedes Lucas M, Delvalle D, Planchot V, Jimenez S, Perez R, Ball S, D'Hulst C, Merida A. The phenotype of soluble starch synthase IV defective mutants of Arabidopsis thaliana suggests a novel function of elongation enzymes in the control of starch granule formation. Plant J. 2007;49(3):492-504.

19. Szydlowski N, Ragel P, Raynaud S, Lucas MM, Roldan I, Montero M, Munoz FJ, Ovecka M, Bahaji A, Planchot V, et al. Starch granule initiation in Arabidopsis requires the presence of either class IV or class III starch synthases. Plant Cell. 2009;21(8):2443-57.

20. Gamez-Arjona FM, Li J, Raynaud S, Baroja-Fernandez E, Munoz FJ, Ovecka M, Ragel P, Bahaji A, Pozueta-Romero J, Merida A. Enhancing the expression of starch synthase class IV results in increased levels of both transitory and long-term storage starch. Plant Biotechnol J. 2011;9(9):1049-60.

21. Crumpton-Taylor M, Pike M, Lu KJ, Hylton CM, Feil R, Eicke S, Lunn JE, Zeeman SC, Smith AM. Starch synthase 4 is essential for coordination of starch granule formation with chloroplast division during Arabidopsis leaf expansion. New Phytol. 2013;200(4):1064-75.

22. Ohdan T, Francisco Jr PB, Sawada T, Hirose T, Terao T, Satoh H, Nakamura Y. Expression profiling of genes involved in starch synthesis in sink and source organs of rice. J Exp Bot. 2005;56(422):3229-44.

23. Toyosawa Y, Kawagoe Y, Matsushima R, Crofts N, Ogawa M, Fukuda M, Kumamaru T, Okazaki Y, Kusano M, Saito K, et al. Deficiency of starch synthase IIIa and IVb alters starch granule morphology from polyhedral to spherical in rice endosperm. Plant Physiol. 2016;170:1255-70.

24. Leterrier M, Holappa LD, Broglie KE, Beckles DM. Cloning, characterisation and comparative analysis of a starch synthase IV gene in wheat: functional and evolutionary implications. BMC Plant Biol. 2008;8:98.

25. Mishra A, Singh A, Sharma M, Kumar P, Roy J. Development of EMS-induced mutation population for amylose and resistant starch variation in bread wheat (Triticum aestivum) and identification of candidate genes responsible for amylose variation. BMC Plant Biol. 2016;16(1):217.

26. Singh A, Kumar P, Sharma M, Tuli R, Dhaliwal HS, Chaudhury A, Pal D, Roy J. Expression patterns of genes involved in starch biosynthesis during seed development in bread wheat (Triticum aestivum). Mol Breed. 2015;35(9):184.

27. Chen L, Huang L, Min D, Phillips A, Wang S, Madgwick PJ, Parry MAJ, Hu Y-G. Development and characterization of a new TILLING population of common bread wheat (Triticum aestivum L.). PLoS One. 2012;7:e41570.

28. Chen A, Jorge D. Wheat TILLING mutants show that the vernalization gene VRN1 down-regulates the flowering repressor VRN2 in leaves but is not essential for flowering. PLoS Genet. 2012;8(12):e1003134.

29. Fitzgerald TL, Kazan K, Li Z, Morell MK, Manners JM. A high-throughput method for the detection of homologous gene deletions in hexaploid wheat. BMC Plant Biol. 2010;10:264.

30. Guo Y, Abernathy B, Zeng Y, Ozias-Akins P. TILLING by sequencing to identify induced mutations in stress resistance genes of peanut (Arachis hypogaea). BMC Genomics. 2015;16(1):157.

31. McCallum CM, Comai L, Greene EA, Henikoff S. Targeting Induced Local Lesions IN Genomes (TILLING) for plant functional genomics. Plant Physiol. 2000;123:439-42.

32. Parry MAJ, Madgwick PJ, Bayon C, Tearall K, Hernandez-Lopez A, Baudo M, Rakszegi M, Hamada W, Al-Yassin A, Ouabbou H, et al. Mutation discovery for crop improvement. J Exp Bot. 2009;60(10):2817-25.

33. Till BJ, Cooper J, Tai TH, Colowit P, Greene EA, Henikoff S, Comai L. Discovery of chemically induced mutations in rice by TILLING. BMC Plant Biol. 2007;7(1):19.

34. Till BJ, Reynolds SH, Greene EA, Codomo CA, Enns LC, Johnson JE, Burtner C, Odden AR, Young K, Taylor NE, et al. Large-scale discovery of induced point mutations with high-throughput TILLING (Arabidopsis). Genome Res. 2003;13:524-30.

35. Dong C, Vincent K, Sharp P. Simultaneous mutation detection of three homoeologous genes in wheat by High Resolution Melting analysis and Mutation Surveyor. BMC Plant Biol. 2009;9:143.

36. Uauy C, Paraiso F, Colasuonno P, Tran RK, Tsai H, Berardi S, Comai L, Dubcovsky J. A modified TILLING approach to detect induced mutations in tetraploid and hexaploid wheat. BMC Plant Biol. 2009;9(1):115.

37. Slade AJ, Fuerstenberg SI, Loeffler D, Steine MN, Facciotti D. A reverse genetic, nontransgenic approach to wheat crop improvement by TILLING. Nat Biotechnol. 2005;23(1):75-81.

38. Hazard B, Zhang X, Colasuonno P, Uauy C, Beckles DM, Dubcovsky J. Induced mutations in the starch branching enzyme II (SBEII) genes increase amylose and resistant starch content in durum wheat. Crop Sci. 2012;52: 1754-66.

39. Bovina R, Brunazzi A, Gasparini G, Sestili F, Palombieri S, Botticella E, Lafiandra D, Mantovani P, Massi A. Development of a TILLING resource in 
durum wheat for reverse- and forward-genetic analyses. Crop Pasture Sci. 2014;65:112-24.

40. Sestili F, Palombieri S, Botticella E, Mantovani P, Bovina R, Lafiandra D. TILLING mutants of durum wheat result in a high amylose phenotype and provide information on alternative splicing mechanisms. Plant Sci. 2015;233: 127-33.

41. Slade AJ, McGuire C, Loeffler D, Mullenberg J, Skinner W, Fazio G, Holm A, Brandt KM, Steine MN, Goodstal JF, et al. Development of high amylose wheat through TILLING. BMC Plant Biol. 2012;12:69.

42. Hazard B, Zhang X, Colasuonno P, Uauy C, Beckles DM, Dubcovsky J. Induced Mutations in the Starch Branching Enzyme II (SBEII) genes increase amylose and resistant starch content in durum wheat. 2012.

43. Shimbata T, Ai Y, Fujita M, Inokuma T, Vrinten P, Sunohara A, Saito M, Takiya T, Jane JL, Nakamura T. Effects of homoeologous wheat starch synthase lla genes on starch properties. J Agric Food Chem. 2012;60(48):12004-10.

44. Ahuja G, Jaiswal S, Hucl P, Chibbar RN. Genome-specific granule-bound starch synthase I (GBSSI) influences starch biochemical and functional characteristics in near-isogenic wheat (Triticum aestivum L.) lines. J Agric Food Chem. 2013;61(49):12129-38.

45. Wickramasinghe HAM, Miura H. Gene dosage effect of the wheat Wx alleles and their interaction on amylose synthesis in the endosperm. Euphytica. 2003:132:303-10.

46. Botticella E, Sestili F, Hernandez-Lopez A, Phillips A, Lafiandra D. High resolution melting analysis for the detection of EMS induced mutations in wheat SBElla genes. BMC Plant Biol. 2011;11:156.

47. Crumpton-Taylor M, Grandison S, Png KM, Bushby AJ, Smith AM. Control of starch granule numbers in Arabidopsis chloroplasts. Plant Physiol. 2012; 158(2):905-16.

48. Hogg AC, Gause K, Hofer P, Martin JM, Graybosch RA, Hansen LE, Giroux MJ. Creation of a high-amylose durum wheat through mutagenesis of starch synthase II (SSIla). J Cereal Sci. 2013;57(3):377-83.

49. Konik-Rose C, Thistleton J, Chanvrier H, Tan I, Halley P, Gidley M, KosarHashemi B, Wang $\mathrm{H}$, Larroque $\mathrm{O}$, Ikea J, et al. Effects of starch synthase lla gene dosage on grain, protein and starch in endosperm of wheat. Theor Appl Genet. 2007;115(8):1053-65.

50. Avni R, Zhao R, Pearce S, Jun Y, Uauy C, Tabbita F, Fahima T, Slade A, Dubcovsky J, Distelfeld A. Functional characterization of GPC-1 genes in hexaploid wheat. Planta. 2014;239(2):313-24.

51. Ludewig F, Sonnewald U, Kauder F, Heineke D, Geiger M, Stitt M, MullerRober BT, Gillissen B, Kuhn C, Frommer WB. The role of transient starch in acclimation to elevated atmospheric $\mathrm{CO}_{2}$. FEBS Lett. 1998;429:147-51.

52. Goldschmidt EE, Huber SC. Regulation of photosynthesis by end-product accumulation in leaves of plants storing starch, sucrose, and hexose sugars. Plant Physiol. 1992;99:1443-8.

53. Ragel P, Streb S, Feil R, Sahrawy M, Annunziata MG, Lunn JE, Zeeman S, Merida A. Loss of starch granule initiation has a deleterious effect on the growth of arabidopsis plants due to an accumulation of ADP-glucose. Plant Physiol. 2013;163(1):75-85.

54. Till BJ, Zerr T, Comai L, Henikoff S. A protocol for TILLING and Ecotilling in plants and animals. Nat Protoc. 2006;1(5):2465-77.

55. Ng PC, Henikoff S. SIFT: predicting amino acid changes that affect protein function. Nucleic Acids Res. 2003;31(13):3812-4.

56. Taylor NE, Greene EA. PARSESNP: a tool for the analysis of nucleotide polymorphisms. Nucleic Acids Res. 2003;31(13):3808-11.

57. Gu J, Wang Q, Cui M, Han B, Guo H, Zhao L, Xie Y, Song X, Liu L. Cloning and characterization of Ku70 and Ku80 homologues involved in DNA repair process in wheat (Triticum aestivum L.). Plant Genet Resourc. 2014;12(S1): S99-S103.

58. Guo H-j, Zhao H-b, Zhao L-s, Gu J-y, Zhao S-r, Li J-h, Liu Q-c, Liu L-x. Characterization of a novel chlorophyll-deficient mutant Mt6172 in wheat. J Integr Agric. 2012:11(6):888-97.

59. Xiong H, Guo H, Xie Y, Zhao L, Gu J, Zhao S, Li J, Liu L. Enhancement of dwarf wheat germplasm with high-yield potential derived from induced mutagenesis. Plant Genet Resourc. 2016. doi:10.1017/S1479262116000459.

\section{Submit your next manuscript to BioMed Central and we will help you at every step:}

- We accept pre-submission inquiries

- Our selector tool helps you to find the most relevant journal

- We provide round the clock customer support

- Convenient online submission

- Thorough peer review

- Inclusion in PubMed and all major indexing services

- Maximum visibility for your research

Submit your manuscript at www.biomedcentral.com/submit
Biomed Central 